\title{
Pharmacogenetic analysis of the mGlu2/3 agonist LY2140023 monohydrate in the treatment of schizophrenia
}

\author{
W Liu ${ }^{1,2}$, ACM Downing ${ }^{1,2}$, \\ LM Munsie ${ }^{1}$, P Chen ${ }^{1}$, \\ MR Reed ${ }^{1}, C^{2}$ Ruble ${ }^{1}$, \\ KT Landschulz ${ }^{1}$, BJ Kinon ${ }^{1}$, \\ LK Nisenbaum ${ }^{1}$
}

${ }^{1}$ Eli Lilly and Company, Indianapolis, IN, USA

\section{Correspondence:}

Dr LK Nisenbaum, Translational Medicine and Pharmacogenomics, Lilly Corporate Center, Eli Lilly and Company, Indianapolis, IN 46285, USA.

E-mail: I.nisenbaum@lilly.com
${ }^{2}$ These two authors contributed equally to this work.

Received 23 April 2010; revised 27 September 2010; accepted 16 November 2010; published online 21 December 2010
The goal of this study was to identify genetic markers associated with LY2140023 monohydrate response in patients with schizophrenia. Variants in eight candidate genes related to the mechanism of action of LY2140023 or olanzapine were investigated in a genetic cohort collected from two clinical trials. Results from this genetic analysis indicate that 23 single nucleotide polymorphisms (SNPs) were associated with a change in Positive and Negative Syndrome Scale total score in response to LY2140023 at 28 days $(P<0.01$; false discovery rate $<0.2)$. Sixteen of these SNPs were located in the serotonin $2 \mathrm{~A}$ receptor (HTR2A). Bioinformatic analyses identified a putative antisense nested gene in intron 2 of HTR2A in the region of the genetic markers associated with LY2140023 response. These data suggest a genetic association exists between SNPs in several genes, such as HTR2A, and response to LY2140023 treatment. Additional clinical trials are needed to establish replication of these results.

The Pharmacogenomics Journal (2012) 12, 246-254; doi:10.1038/tpj.2010.90; published online 21 December 2010

Keywords: schizophrenia; pharmacogenetics; metabotropic glutamate receptor agonist; HTR2A; NRG1; antipsychotics

\section{Introduction}

Schizophrenia, a chronic and disabling psychiatric disorder, affects $\sim 1 \%$ of adults worldwide. ${ }^{1}$ Although the underlying etiology remains unknown, dysregulation of dopaminergic and serotonergic neurotransmission is considered a contributing factor to the pathophysiology of schizophrenia. ${ }^{2}$ Blockade of dopamine and/or serotonin receptors has been the primary focus in the management of schizophrenia. Despite treatment with antipsychotic agents, $40-50 \%$ of patients do not adequately respond, ${ }^{3}$ resulting in relapse, poor prognosis, psychiatric and medical complications, and death from medical comorbidities or suicide. ${ }^{4}$ Optimizing response by individualizing antipsychotic treatment is key to achieving successful outcomes in patients with schizophrenia.

Evidence from several clinical and preclinical studies supports the hypothesis that dysregulation of glutamatergic pathways contributes to the pathogenesis of schizophrenia. ${ }^{5-7}$ LY404039 is a potent and highly selective agonist for group II metabotropic glutamate receptors (mGlu2 and mGlu3 receptors). LY2140023 monohydrate is an oral methionine prodrug of LY404039. LY2140023 is currently being investigated as an antipsychotic agent with a novel mechanism 
of action. Used herein, LY2140023 refers to the monohydrate form of the oral prodrug of LY404039. In a phase II proof-of-concept trial (H8Y-BD-HBBD (HBBD)), LY2140023 demonstrated significant improvement in Positive and Negative Syndrome Scale (PANSS) total score compared with placebo. ${ }^{8}$ These findings suggest that mGlu2/3 receptor agonists may be effective for the treatment of schizophrenia.

Genetic factors are theorized to have an important role in antipsychotic treatment response; ${ }^{9}$ however, contributions of specific genetic variants remain poorly understood. ${ }^{10}$

Candidate gene and genome-wide association studies of treatment response to typical and atypical antipsychotics have identified multiple genes associated with response, ${ }^{11}$ though replication of these findings has not been consistent. ${ }^{10}$ Continued research to understand the impact of specific genetic variants on individual patient response is required. The primary goal of this pharmacogenetic study was to identify genetic markers uniquely associated with LY2140023 response. Using DNA samples collected in two clinical trials, the association between single nucleotide polymorphisms (SNPs) and LY2140023, placebo or olanzapine response was examined in eight genes related to antipsychotic mechanism of action or schizophrenia disease susceptibility, including mGlu2 receptor (GRM2), mGlu3 receptor (GRM3), serotonin 2A receptor (HTR2A), dopamine receptor D2 (DRD2), dopamine receptor D3 (DRD3), neuregulin 1 (NRG1), catechol-O-methyltransferase (COMT) and polycystic kidney and hepatic disease 1 (PKHD1).

\section{Materials and methods}

\section{Study population}

DNA samples were collected in two clinical trials, a phase II proof-of-concept trial (HBBD), which assessed LY2140023 efficacy compared with olanzapine and placebo in patients with schizophrenia, ${ }^{8}$ and a phase IV fixed dose-response clinical trial (F1D-US-HGLF (HGLF)), which compared efficacy of olanzapine at one of three dosages: 10, 20 or $40 \mathrm{mg}$ QD in patients with schizophrenia or schizoaffective disorder. ${ }^{12}$ Study designs and clinical findings of both trials have been published. ${ }^{8,12}$ The HBBD study was conducted at 10 sites in Russia. All patients had a confirmed diagnosis of schizophrenia as assessed by a modified Structured Clinical Interview for Diagnostic and Statistical Manual of Mental Disorders IV (DSM-IV) Axis I Disorders and were hospitalized for the duration of study participation. All patients selfreported their ethnicity as Caucasian. The HGLF study was conducted at 55 study sites in the United States. Both inpatients and outpatients with a diagnosis of DSM-IV schizophrenia or schizoaffective disorder were eligible for trial participation. Only the self-reported Caucasians were eligible for the pharmacogenetic analysis to avoid ethnicity confounds. Both studies used PANSS total score as the primary endpoint and were approved by participating sites institutional review boards; all patients provided written informed consent. The majority of DNA samples included in the current pharmacogenetic study were obtained from patients in the HBBD trial; however, as the olanzapine treatment arm had half the number of patients as the placebo and LY2140023 arms, DNA samples collected from patients treated with olanzapine in the HGLF trial were included in this pharmacogenetic study to increase the sample size in this treatment group.

\section{Genotyping and SNP selection}

A relatively small number of candidate genes was investigated due to the limited power of the study (see Statistical analysis section). Genes were selected for inclusion based on several criteria. GRM2 and GRM3 are the known pharmacologic targets of the mGlu agonist, LY404039, ${ }^{13}$ while DRD2 and HTR2A are among the primary pharmacological targets of olanzapine. NRG1 and COMT have both been implicated as schizophrenia disease susceptibility genes, with NRG1 related to glutamate signaling and COMT related to dopaminergic activity. Finally, a limited number of SNPs within PKHD1 were included based on the association of this gene with atypical antipsychotic treatment-emergent weight gain. ${ }^{14-17}$

Genotyping was conducted on two SNP panels. SNPs selected for the two panels covered the same set of candidate genes: GRM2, GRM3, HTR2A, DRD2, DRD3, NRG1, COMT and PKHD1 (PKHD1 was excluded from Panel 2). Panel 2 genotyping was conducted to add redundancy and to supplement coverage from Panel 1 assay failures. From within each of the candidate genes (PKHD1 excluded) and including 5000 bp of flanking sequence, SNP selection was performed utilizing the dbSNP database from National Center for Biotechnology Information with a combination of criteria as follows: (1) all tagging SNPs, (2) all nonsynonymous SNPs, (3) synonymous SNPs with minor allele frequency (MAF) $>2 \%$ or unreported MAF and (4) noncoding region SNPs with MAF $>20 \%$. The rationale for these criteria was to provide extensive coverage of the genes for redundancy, potential assay failures and the ability to determine linkage disequilibrium (LD) across this population of patients. In addition, SNPs within GRM3 that had previously been associated with schizophrenia disease susceptibility, endophenotypes or antipsychotic treatment response were also included. ${ }^{18-20}$ The number of SNPs analyzed within each gene can be found in Supplementary Table 1.

\section{Genotyping quality control}

Genotyping was performed by Sequenom (San Diego, CA, USA) using the MassARRAY platform as previously described. ${ }^{21}$ Of the 200 DNA samples sent for genotyping, genotype data from 196 patients were generated for Panel 1 SNPs (Supplementary Figure 1). One sample genotyped on Panel 1 had a 0\% call rate and was not resent for Panel 2 genotyping. Genotype data from 199 patients were generated for Panel 2 SNPs (Supplementary Figure 1).

Samples with call rates $\leqslant 95 \%$ or a $\geqslant 9 \%$ discordant rate within assay quality control duplicate SNPs were excluded from the analysis, as were monomorphic SNPs, SNPs with call rates $\leqslant 95 \%$, MAF $>2 \%$ or Hardy-Weinberg equilibrium 
$P<0.001$. A total of 488 and 92 SNPs comprised the final Panel 1 and Panel 2 genotype data sets, respectively.

\section{Statistical analysis}

The pre-defined primary analysis tested the association between genetic variants and clinical response to LY2140023 as measured by the PANSS total score after 28 days of treatment by using a last-observation-carriedforward (LOCF) model on the intent-to-treat population. The association between genetic variants and placebo/ olanzapine treatment response was also tested in an exploratory analysis. Changes in PANSS total score were modeled using analysis of covariance. The dependent variable was change in PANSS total score from baseline; independent variables were gender, baseline PANSS total score, age at trial entry, trial (HBBD vs HGLF), assigned study treatment, genotype and treatment-by-genotype interaction, assuming additive inheritance. Linear contrast was used to estimate genotype effect on response for each SNP separately within each treatment group. Multiple comparison adjustments were carried out independently within each treatment arm for the two panels as the analyses were conducted separately, with $P$-values converted into false discovery rate (FDR). ${ }^{22}$

Based on 66 patients available in the LY2140023 treatment arm, power was calculated assuming additive inheritance using nQuery Advisor 7.0 (Statistical Solutions, Farmers Cross, Cork, Ireland). Use of these assumptions yielded $67 \%$ power to detect a 10.4-point change in PANSS total score at a significance level of $P<0.01$.

Mixed-effects-repeated-measures model (MMRM) analysis was conducted within the LY2140023 arm for sensitivity. The dependent variable, change in PANSS total score from baseline, was fit to a model with baseline PANSS total score, gender, genotype, visit and genotype-by-visit interaction terms. A genotypic model was assumed where the genotype was treated as a categorical variable in the MMRM analysis.

Linkage disequilibrium and haplotype block analyses were conducted using Haploview 4.1 (Broad Institute, Cambridge, MA, USA). ${ }^{23}$ Association between genotype and secondary efficacy measures, including PANSS-positive, PANSS-negative and clinical global impression-severity scores were tested using an LOCF model similar to the one used in the primary PANSS total score analysis. SAS 9.1 was used for all analyses (SAS Institute, Cary, NC, USA).

\section{Antisense nested gene}

Bioinformatic analysis of HTR2A SNP-containing intronic sequence. The genomic sequence ref|NC_000013.9|NC_ 000013:c46378176-46295514 was masked using RepeatMasker (http://repeatmasker.org) then used as a BLAST query against est_human and nt (http://ftp://ftp.ncbi.nih.gov/ blast $/ \mathrm{db} /$ ). Hits were mapped onto the genomic sequence using gmap software (http://research-pub.gene.com/gmap/). Splice form-specific contigs were created using Sequencher (Gene Codes Corporation, Ann Arbor, MI, USA). The Artemis software (http://www.sanger.ac.uk/Software/Artemis/) and
BioPerl modules (http://www.bioperl.org) were used to visualize and annotate the genomic sequence.

Detection and characterization of the antisense transcript. Reverse transcription polymerase chain reaction (RT-PCR) for the antisense nested gene was performed using RNA from human testes and frontal cortex (Catalog \#AM7972 and \#AM6810, First Choice Total RNA; Ambion, Austin, TX, USA). Two micrograms of RNA was reverse transcribed into cDNA using Qiagen's OneStep RT-PCR kit (Qiagen, Hilden, Germany) according to the manufacturer's protocol. In a denaturation step, reverse transcription primer and RNA were mixed and incubated at $65^{\circ} \mathrm{C}$ for $5 \mathrm{~min}$ before adding the remaining reagents. Primers were designed to sequences within intron 2 of HTR2A corresponding to exons 1 and 3 of the antisense nested gene. Complementary to the sequence in exon 3 of the antisense nested gene, the primer 5'-CAAGGAAGATGTACTTGAATCTTCTCC- ${ }^{\prime}$ was used for the reverse transcription step. A second primer 5'-CAGAAAGAACGCAGCCCTAC-3', targeting exon 1 of the antisense nested gene, was added after the initial PCR activation step. At an annealing temperature of $55^{\circ} \mathrm{C}$, PCR was performed for 50 cycles, followed by a second round of 50 cycles of PCR at a 1:100 dilution of the Qiagen OneStep RT-PCR reaction using antisense nested gene exon 1 primer $5^{\prime}$-CCAACTTTGGACTTTTCACTTCCAA- ${ }^{\prime}$ and exon 3 primer $5^{\prime}$-ATCTTCTCCACCCACTGTCC-3'. These primers, located interior to the primer pair, were used for the first round of PCR products and served to amplify low-level RNA species. To confirm band identity, PCR products were run on $2 \%$ agarose gels and sequenced under standard conditions (Agencourt, Beverly, MA, USA). Additionally, IMAGE cDNA clones representing antisense nested gene transcripts were obtained (Thermo Scientific Open Biosystems, Huntsville, AL, USA) and sequenced for identification of additional 5'exons.

\section{Results}

\section{Characteristics of the patient population}

Caucasian patients with a baseline and at least one postbaseline PANSS total score were eligible for inclusion in the pharmacogenetics study. DNA samples from 193 eligible patients (HBBD, $n=139$ and HGLF, $n=54$ ) met either the first or second panel genotyping quality control criteria and were designated as the genetic cohort (Supplementary Figure 1).

Patients from the genetic cohort demonstrated similar demographics and baseline PANSS total scores compared with the HBBD clinical trial population (Table 1). Analyses conducted on Panel 1 (488 SNPs) included 180 patients (LY2140023, $n=66$; olanzapine, $n=73$ and placebo, $n=41$ ). Analyses performed on Panel 2 (92 SNPs) included 169 patients (LY2140023, $n=63$; olanzapine, $n=69$ and placebo, $n=37$ ). Pairwise LD analysis was conducted on 157 Caucasian patients whose DNA samples passed genotyping quality control from both panels. 
Table 1 Baseline characteristics for HBBD clinical trial population and HBBD/HGLF genetic cohort

\begin{tabular}{|c|c|c|c|c|c|c|c|}
\hline Trial & $\mathrm{N}$ & Mean PANSS total score & Therapy & $\mathrm{n}$ & Age & PANSS total & Female \% \\
\hline \multirow[t]{2}{*}{ HBBD (clinical trial population) } & 195 & 94.8 & Placebo & 63 & 41.0 & 93.8 & 22.2 \\
\hline & & & LY2140023 & 98 & 39.6 & 95.5 & 20.4 \\
\hline \multirow[t]{3}{*}{ HBBD+HGLF (genetic cohort) } & 193 & 93.2 & Placebo & 43 & 40.9 & 93.0 & 20.9 \\
\hline & & & LY2140023 & 71 & 40.3 & 94.6 & 25.4 \\
\hline & & & $\mathrm{Olz}$ & $79^{a}$ & 41.9 & 91.9 & 38.0 \\
\hline
\end{tabular}

Abbreviations: Olz, olanzapine; PANSS, Positive and Negative Syndrome Scale.

${ }^{a} 25$ from HBBD and 54 from HGLF.

Table 2 SNPs associated with change in PANSS total score $(P<0.01)$ in response to LY2140023 treatment

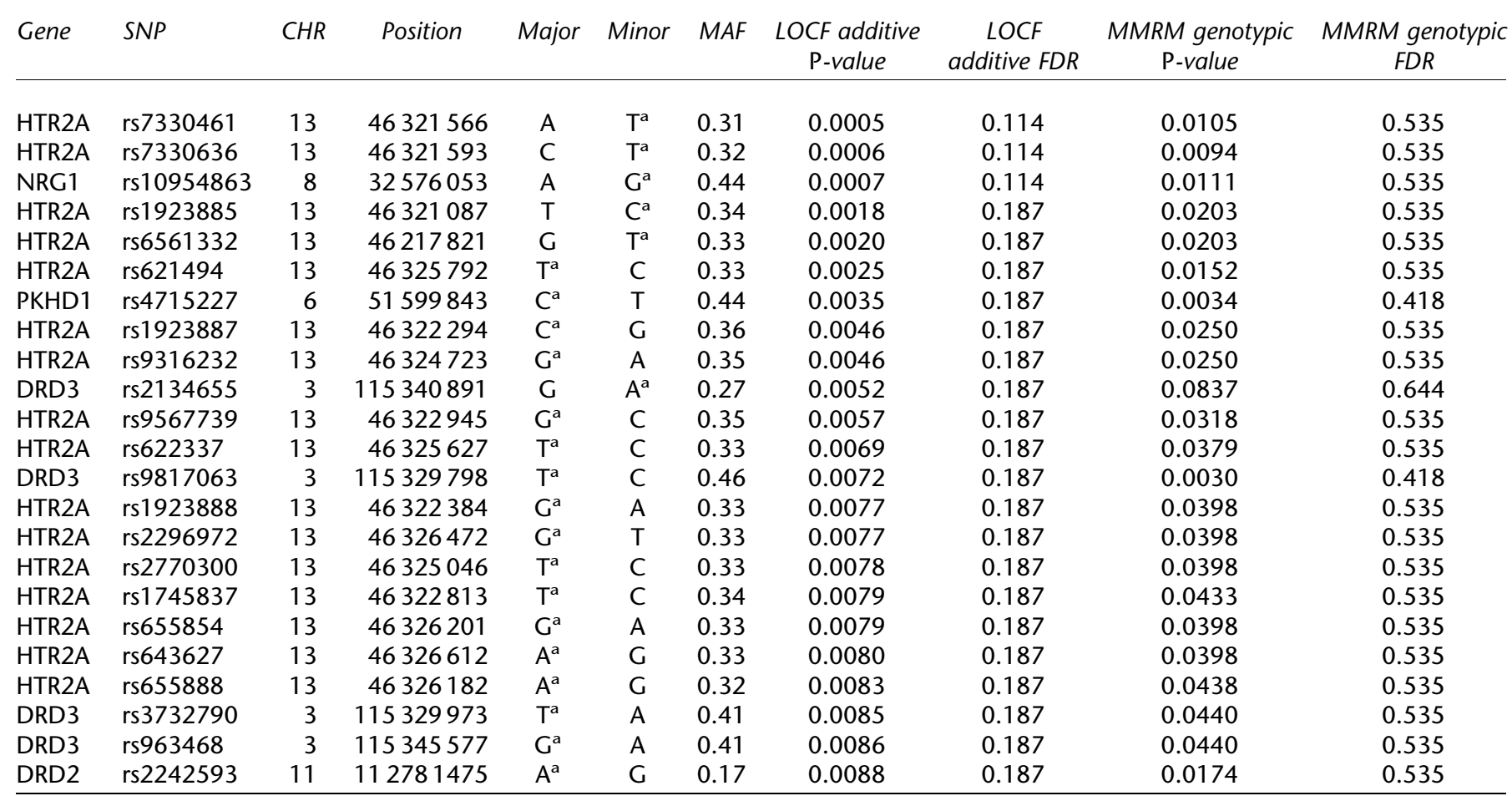

Abbreviations: CHR, chromosome; DRD2, dopamine receptor D2; DRD3, dopamine receptor D3; FDR, false discovery rate; HTR2A, serotonin 2A receptor; LOCF, lastobservation-carried-forward; MAF, minor allele frequency; MMRM, mixed-effects-repeated-measures model; NRG1, neuregulin 1; PANSS, Positive and Negative Syndrome Scale; PKHD1, polycystic kidney and hepatic disease 1; SNP, single nucleotide polymorphism.

aLY2140023-responsive allele.

\section{Primary efficacy measure}

LOCF analysis with additive allelic assumption. Twenty-three SNPs were associated with change in PANSS total score at 28 days in the LY2140023 treatment group with an unadjusted $P<0.01$ (FDR $<0.2$; Table 2), but showed no association in the olanzapine or placebo arms. Of these 23 SNPs, three (rs7330461 and rs7330636 in HTR2A, and rs10954863 in NRG1) were associated with LY2140023 response with an unadjusted $P<0.001$ (FDR $=0.114)$. The HTR2A SNPs were separated by $27 \mathrm{bp}$ and were in strong $\mathrm{LD}\left(D^{\prime}=0.97\right)$. The minor homozygous genotype group showed the largest reduction in PANSS total score for each of the three SNPs, whereas the major homozygous genotype group showed the smallest reduction. In contrast, the response profile for the three SNPs showed no association across genotype groups for either olanzapine or placebo. Figure 1 illustrates change in PANSS total score as a function of genotype for one of the two tightly linked SNPs in HTR2A, rs7330461, and the NRG1 SNP, rs10954863.

All 23 SNPs associated with LY2140023 response $(P<0.01)$ were located within non-coding regions of their respective genes; 16 SNPs were located within two haplotype blocks in HTR2A. All 16 were in relatively high $\mathrm{LD}\left(D^{\prime}>0.75\right)$, with two SNPs, rs6561332 and rs1923885, located within block 

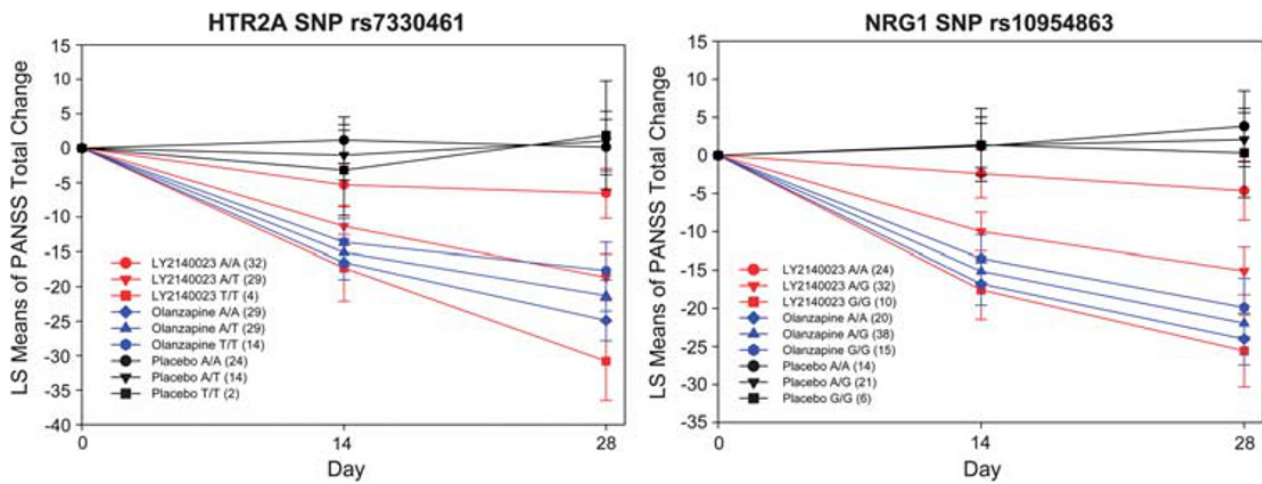

Figure 1 Effect of HTR2A SNP rs7330461 and NRG1 SNP rs10954863 genotypes on change in Positive and Negative Syndrome Scale total score (last-observation-carried-forward). Number of patients in each genotype group is represented in parentheses. HTR2A, serotonin 2A receptor; LS, least squares; NRG1, neuregulin 1; PANSS, Positive and Negative Syndrome Scale; SNP, single nucleotide polymorphism.

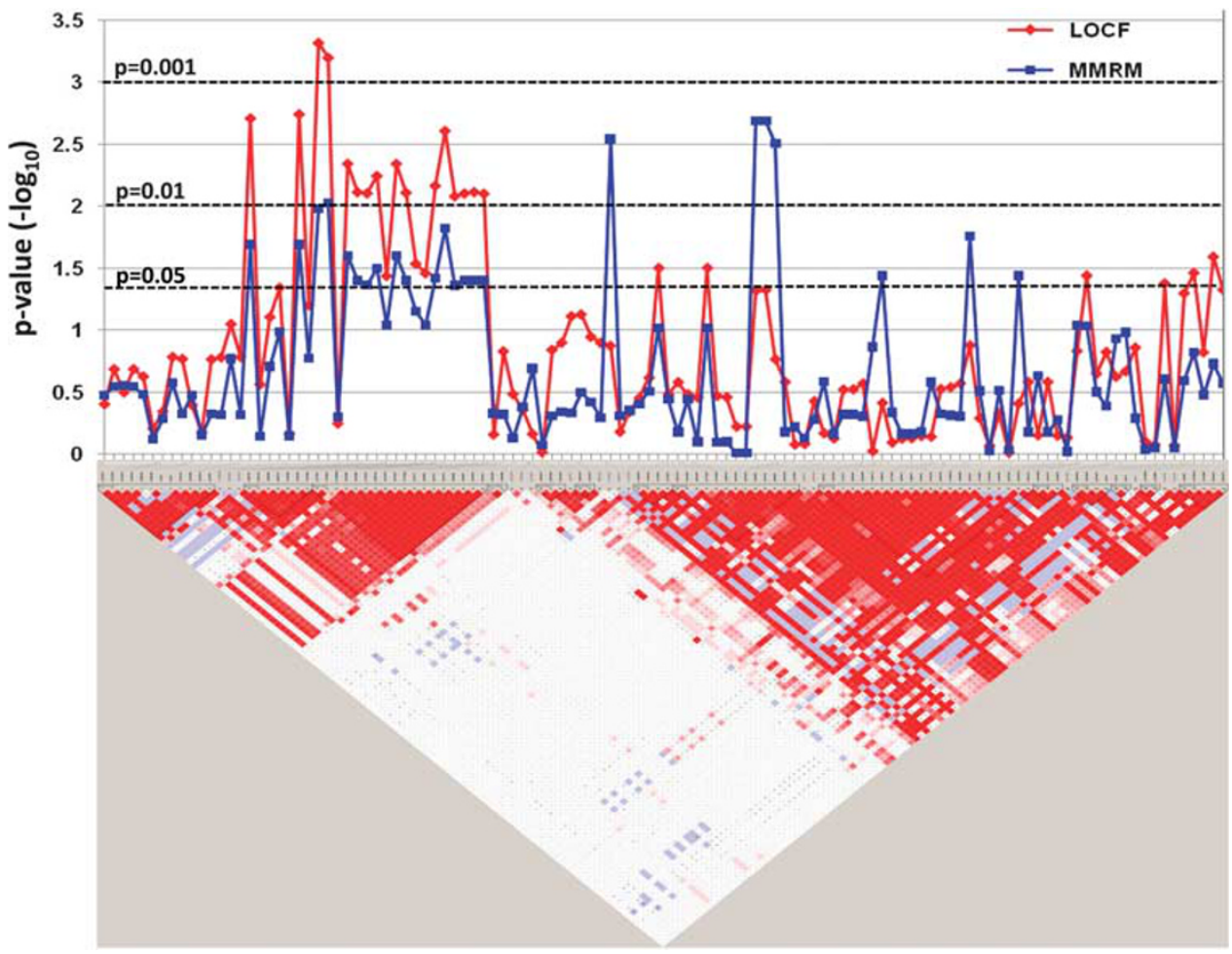

Figure 2 HTR2A: LD, haplotype blocks, and $P$-values associated with change in PANSS total score for each SNP genotyped. (Top panel) -log 10 transformed $P$-values of change in PANSS total score by analysis model (LOCF vs MMRM) for each SNP. (Lower panel) Pairwise $D^{\prime} \times 100$ and the haplotype blocks. The standard Haploview color scheme is used for LD color display (LOD score $\geqslant 2$ and $D^{\prime}=1$ in bright red; LOD score $\geqslant 2$ and $D^{\prime}<1$ in shades of pink/red; LOD score $<2$ and $D^{\prime}=1$ in blue; LOD score $<2$ and $D^{\prime}<1$ in white). LD, linkage disequilibrium; LOCF, last-observation-carriedforward; LOD, logarithm of odds; MMRM, mixed-effects-repeated-measures model; PANSS, Positive and Negative Syndrome Scale; SNP, single nucleotide polymorphism.

2 and the remaining 14 residing in block 3 (Figure 2). Of the remaining SNPs, four were in DRD3, one in PKHD1 and one in DRD2 (Table 2).

Within the olanzapine arm, one NRG1 SNP, rs34861396, was associated with change in PANSS total score at 28 days (unadjusted $P<0.01, F D R=0.95$ ). This NRG1 SNP was $958 \mathrm{~kb}$ pairs away from rs10954863, the NRG1 SNP associated with LY2140023 response. Within the placebo arm, one HTR2A SNP, rs3125, was associated with change in
PANSS total score at 28 days (unadjusted $P<0.01, \mathrm{FDR}=1$ ). This HTR2A SNP was $15 \mathrm{~kb}$ away from rs7330461, one of the HTR2A SNPs associated with LY2140023. Neither of the SNPs associated with olanzapine nor placebo response was in strong LD $\left(D^{\prime}<0.55\right)$ with the LY2140023associated SNPs.

MMRM analysis with genotypic assumption. The 23 LY2140023-associated SNPs identified in the LOCF analysis 
also showed similar effects in the MMRM sensitivity analysis. At 28 days, 22 of the 23 SNPs were associated with change in PANSS total score (unadjusted $P<0.05$; Table 2). The change in PANSS total score in MMRM analysis for the HTR2A SNP, rs7330461, is shown in Supplementary Figure 2. Consistent with the primary LOCF results, the minor homozygous genotype group remained the most responsive, whereas the major homozygous genotype group was the least responsive.

\section{Secondary efficacy measures}

The secondary efficacy results, including changes in PANSSpositive, PANSS-negative and clinical global impressionseverity scores, were similar to the primary efficacy results for the 23 LY2140023-associated SNPs (Supplementary Table 2). The change in secondary efficacy measures as a function of genotype for the HTR2A SNP, rs7330461, is illustrated in Supplementary Figure 3.

A total of 21 SNPs in DRD2 reached significance for change in PANSS-negative score $(P<0.01$; Supplementary

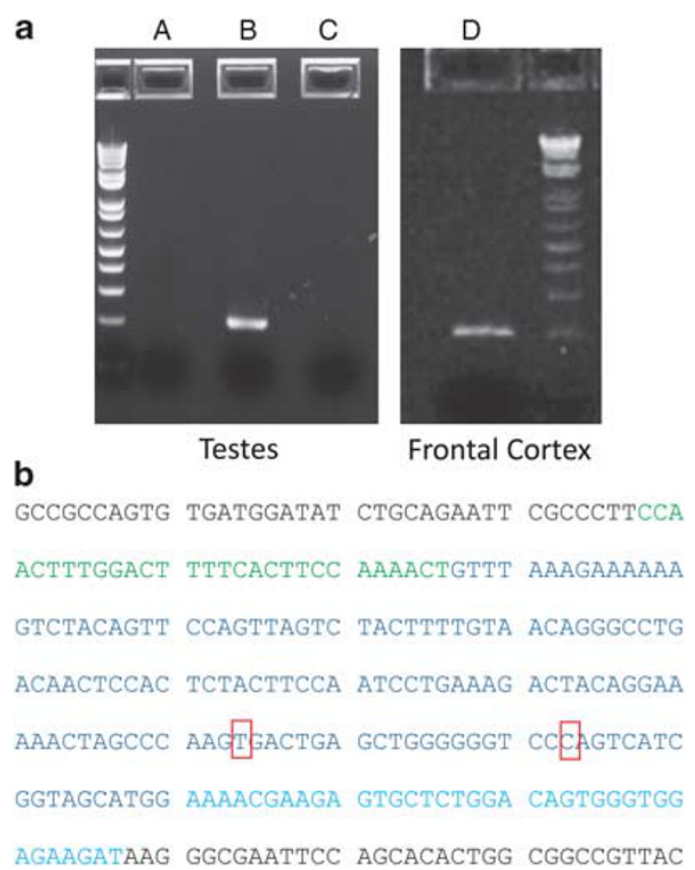

Figure 3 Amplification of antisense transcripts containing nested exons 1, 2 and 3 in human testes and frontal cortex. (a) Strand-specific RT-PCR demonstrating expression of the transcript from the antisense strand (lane B) but not the HTR2A strand (lane A) in total testes RNA (left panel). The product of reaction run in absence of reverse transcriptase is shown in lane C. Conventional RT-PCR demonstrating expression of antisense transcript in total frontal cortex RNA (lane D; right panel). HTR2A, serotonin 2A receptor; RT-PCR, reverse transcription polymerase chain reaction. (b) Sequence obtained from frontal cortex PCR amplification product confirming antisense spliced sequence. Antisense exon 1 sequence is shown in green, exon 2 sequence is shown in blue and exon 3 sequence is shown in cyan text. Priming vector is indicated by black text. Red boxes indicate the location of two SNPs associated with LY2140023 response present within antisense exonic regions. PCR, polymerase chain reaction; SNPs, single nucleotide polymorphisms.
Table 3). Nineteen of these DRD2 SNPs were also associated with change in PANSS total score $(P<0.05)$. These 21 DRD2 SNPs were located within two haplotype blocks and were all in high LD $\left(D^{\prime} \geqslant 0.95\right)$ (Supplementary Figure 4$)$. Other secondary measures examined did not uncover additional signals warranting further investigation.

Evidence of antisense nested gene within HTR2A intron 2. Given the large number of associated SNPs located within intron 2 of HTR2A, bioinformatic analyses were conducted to determine whether any functional elements were contained within this intronic region. Although no functional elements were detected on the HTR2A strand of DNA, cDNA and expressed sequence tag database mining identified a putative antisense nested gene in this intronic region colocalizing with the genetic markers associated with LY2140023 response (Supplementary Figure 5). Expressed sequence tags corresponding to antisense nested gene exons were detected in fetal lung, testes and B-cell cDNA libraries. Subsequently, sequence confirmation of IMAGE clones representing these expressed sequence tags yielded expected exons 1, 2 and 3, an alternative exon 2.1, and an additional upstream exon designated as 0a (Supplementary Figure 5). Using RT-PCR and DNA sequencing, the existence of the antisense nested gene transcript in both testes and frontal cortex total RNA was confirmed (Figure 3). Antisense directionality was underscored by the consistent use of consensus donor/acceptor splice sites for each exon-intron boundary, and was additionally supported by strand-specific PCR amplification in which the nested transcript was only observed with antisense directed priming. A transcript length of 475 nucleotides was confirmed, with a low (42\%) guanine/cytosine content. The longest open reading frame in the transcript is 20 amino acids, with no recognized homology to known proteins or RNAs. Two of the 16 HTR2A SNPs associated with LY2140023 response fall within exon 2 of the antisense transcript, while the remaining SNPs are located within intronic regions.

\section{Discussion}

This pharmacogenetic study is the first to examine the influence of genetic variants on response to an mGlu2/3 receptor agonist for the treatment of schizophrenia. Three SNPs (one in NRG1 and two in HTR2A) were associated with LY2140023 response as measured by change in PANSS total score from baseline after 28 days with $P<0.001$ and $\mathrm{FDR}=0.114$. An additional 20 SNPs (14 in HTR2A, 4 in DRD3, 1 in PKHD1 and 1 in DRD2) were found to be associated with LY2140023 response with $P<0.01$ and FDR $<0.2$. Although 8 candidate genes were examined, 16 of the 23 SNPs associated with response $(P<0.01)$ were within the HTR2A gene. Based on response and LD patterns, all 16 HTR2A SNPs may be associated with one causal locus and thus may reflect the same genetic signal. A 30-point PANSS total reduction was seen in individuals in the most responsive genotype group for HTR2A SNP, rs7330461. This 
reduction is three times greater than the reduction in the least responsive genotype group and numerically greater than the mean improvement of patients treated with olanzapine, irrespective of genotype.

In addition to the SNPs identified in the PANSS total analysis, 20 additional DRD2 SNPs were associated with change in PANSS-negative score at 28 days within the LY2140023 arm $(P<0.01)$. The stronger association of these SNPs with PANSS-negative subscale than PANSS total may suggest specificity in improvement of this difficult-to-treat symptom domain for selected genotypes. Interestingly, all of the associated DRD2 SNPs were in high LD and may be reflective of the same causal locus.

In addition to the single marker analysis, four gene-based tests (Fisher's combination test, Sidak's combination test, Simes' combination test and the FDR method) outlined in Peng et al. ${ }^{24}$ were conducted across the analyzed SNPs. The smallest gene-based $P$-values across all four tests were obtained for HTR2A (Supplementary Table 1), suggesting that of the SNPs analyzed, the most likely to be associated with LY2140023 treatment response are contained within this gene. NRG1 had the second smallest $P$-values in Sidak's combination and FDR tests while DRD2 had the second smallest $P$-values in Fisher's combination test, suggesting that SNPs within these genes are also likely to be associated with LY2140023 treatment response.

All SNPs associated with LY2140023 response (Table 2) are located within non-coding regions of their respective genes; thus, the molecular mechanism underlying differential treatment response is not immediately apparent. Despite the lack of known function of the specific SNPs, the biological relevance of the candidate genes is established. If the LY2140023-associated SNPs affect expression of their respective genes, a plausible hypothesis underlying differential LY2140023 treatment response can be proposed. For example, NRG1 has been implicated in glutamatergic signaling and synaptic plasticity via ErbB4 stimulation. ${ }^{16}$ Genetic variants in NRG1 have been implicated in schizophrenia disease susceptibility, suggesting that altered NRG1 expression may contribute to disease symptomatology. ${ }^{25,26}$ Although the LY2140023-associated NRG1 SNP, rs10954863, is not in tight LD with SNPs previously associated with schizophrenia, it is possible that altered NRG1 expression could produce differential clinical LY2140023 response by modulating glutamatergic signaling.

Similar to the atypical antipsychotics olanzapine and clozapine, mGlu2/3 receptor agonists modulate dopamine release and turnover in the brain. ${ }^{27,28}$ Therefore, altered expression of dopamine receptors could lead to differential clinical response to mGlu2/3 receptor agonists. Several of the DRD2 SNPs associated with LY2140023 response have been shown to influence splicing of the dopamine D2 receptor into the D2L (long) and D2S (short) forms. ${ }^{29}$ In addition, these SNPs influence cognitive function and physiological response in healthy humans. ${ }^{29,30}$ The degree of D2 receptor splicing influences dopaminergic modulation of glutamate transmission; ${ }^{31,32}$ therefore, the alterations in D2 receptor splicing associated with DRD2 SNPs may provide greater opportunity for LY2140023 to alter glutamate activity, leading to enhanced treatment response.

The actions of mGlu2/3 receptor agonists involve suppression of excitatory limbic serotonin neurotransmission mediated through HTR2As. ${ }^{33}$ Moreover, recent data suggest that mGlu2 and HTR2As form functional complexes in the brain, which may be involved in altered function in schizophrenia. ${ }^{34}$ Thus, altered expression of HTR2A could underlie differential response to mGlu2/3 receptor agonists. Based on the large number of LY2140023-associated SNPs located within HTR2A intron 2, we examined this region of the gene for potential regulatory elements. Using bioinformatic analyses, we identified an antisense nested gene located in intron 2 of HTR2A. Its characteristics, including its small size, low guanine/cytosine content, short open reading frame and no homology to known proteins or RNAs, suggest it is likely a non-coding RNA.

Only recently has a widespread expression of non-coding antisense RNA been recognized. ${ }^{35,36}$ Various functions of these transcripts are still being elucidated. Some studies suggest that antisense transcripts have a role in the direct, post-transcriptional regulation of RNA levels of the sense transcript, while others demonstrate an epigenetic role in chromatin modification. ${ }^{37}$ Nonetheless, non-coding antisense transcripts often appear to regulate expression levels of the corresponding sense transcript. Our data provide evidence for a non-coding antisense RNA expressed from the same region of HTR2A intron 2 as the 16 LY2140023associated SNPs, suggesting the possibility that genetic variants may exert their effect on response through the regulation of the antisense nested gene. The association and known interactions between the serotonergic and glutamatergic systems warrant continued exploration. Further investigation will be needed to explore the link between the observed SNPs and the antisense nested gene, as well as the antisense nested gene and HTR2A expression.

This study has several strengths. First, the effect size of many of the associated SNPs is relatively large, increasing the potential to use these SNPs as clinically meaningful genetic biomarkers. Second, SNPs associated with significant response $(P<0.001)$ have MAFs ranging between 0.3 and 0.45 in the Caucasian genetic cohort, suggesting that a substantial number of patients may benefit from LY2140023 treatment, assuming the genetic association results are consistently replicated. Third, SNPs associated with LY2140023 response did not demonstrate association with olanzapine or placebo, suggesting a potentially treatmentspecific effect. Fourth, results obtained using different statistical models were similar. Finally, SNPs associated with LY2140023 response displayed consistent results across different efficacy measures, validating the association, particularly as clinical global impression-severity correlates highly with PANSS. ${ }^{38}$

These results should be interpreted with caution until replication is achieved. As the development of LY2140023 continues, additional DNA samples will be collected to further investigate the impact of genetic variation on treatment response. The validity of the findings beyond a 
Caucasian population is uncertain, as known ethnic differences in genotype and allele frequencies exist for many LY2140023-associated SNPs. Another potential limitation of the study was the supplementation of the olanzapine arm with patients from the HGLF study. Unlike HBBD, which was conducted in Russian patients with schizophrenia, HGLF examined patients in the United States diagnosed with either schizophrenia or schizoaffective disorder. The additional HGLF patients may have introduced heterogeneity into the study population and diluted the genetic effect in the olanzapine arm. However, this confound should not influence the associations observed in the LY2140023 arm.

The ability to personalize treatment in psychiatry based upon genotype has been elusive to date. The potential benefits coupled with the novel mechanism of action of LY2140023 clearly call for additional investigation into individualized treatment options. If the clinical effect of LY2140023 is confirmed and the genetic association is successfully replicated, the impact to patients could be substantial.

\section{Conflict of interest}

At the time of the study and during the development of the manuscript, all authors were employees and/or shareholders of Eli Lilly and Company. W Liu, MR Reed and KT Landschulz are currently not employees of Eli Lilly and Company. Funding for this investigation was provided by Eli Lilly and Company, which was also responsible for the study designs; the collection, analysis, and interpretation of data; the writing of the report; and the decision to submit the paper for publication.

\section{Acknowledgments}

We acknowledge the contributions of Duytrac Nguyen, MS, Stewart Fossceco, PhD, Lei Shen, PhD, Chaehyung Ahn, PhD and Sindee Sutherland, PhD, for statistical support; Jeffrey S Arnold, MS, and John N Calley, PhD, for bioinformatic support; Reuben Njau, MS, and John E Brandt, PhD, for operational support; and Sylvia Hanna, $\mathrm{MD}$, employee of i3Statprobe, for writing support. We also thank the study participants without whom this study would not have been possible.

\section{References}

1 Rossler W, Salize HI, van Os J, Riecher-Rossler A. Size of burden of schizophrenia and psychotic disorders. Eur Neuropsychopharmacol 2005; 15: 399-409.

2 Rogers DP, Goldsmith CA. Treatment of schizophrenia in the 21st Century: beyond the neurotransmitter hypothesis. Expert Rev Neurother 2009; 9: 47-54.

3 Lacro JP, Dunn LB, Dolder CR, Leckband SG, Jeste DV. Prevalence of and risk factors for medication nonadherence in patients with schizophrenia: a comprehensive review of recent literature. J Clin Psychiatry 2002; 63: 892-909.

4 Kane JM. Utilization of long-acting antipsychotic medication in patient care. CNS Spectr 2006; 11: 1-7.

5 Krystal JH, D'Souza DC, Petrakis IL, Belger A, Berman RM, Charney DS et al. NMDA agonists and antagonists as probes of glutamatergic dysfunction and pharmacotherapies in neuropsychiatric disorders. Harv Rev Psychiatry 1999; 7: 125-143.
6 Lahti AC, Weiler MA, Tamara Michaelidis BA, Parwani A, Tamminga CA. Effects of ketamine in normal and schizophrenic volunteers. Neuropsychopharmacology 2001; 25: 455-467.

7 Mouri A, Noda Y, Enomoto T, Nabeshima T. Phencyclidine animal models of schizophrenia: approaches from abnormality of glutamatergic neurotransmission and neurodevelopment. Neurochem Int 2007; 51: 173-184.

8 Patil ST, Zhang L, Martenyi F, Lowe SL, Jackson KA, Andreev BV et al. Activation of mGlu2/3 receptors as a new approach to treat schizophrenia: a randomized Phase 2 clinical trial. Nat Med 2007; 13: 1102-1107.

9 Arranz MJ, Kapur S. Pharmacogenetics in psychiatry: are we ready for widespread clinical use? Schizophr Bull 2008; 34: 1130-1144.

10 Patnaik M, Renda MJ, Athanasiou MC, Reed CR. The role of pharmacogenetics in treating central nervous system disorders. Exp Biol Med (Maywood) 2008; 233: 1504-1509.

11 Blanc O, Brousse G, Meary A, Leboyer M, Llorca PM. Pharmacogenetic of response efficacy to antipsychotics in schizophrenia: pharmacodynamic aspects. Review and implications for clinical research. Fundam Clin Pharmacol 2010; 24: 139-160.

12 Kinon BJ, Volavka J, Stauffer V, Edwards SE, Liu-Seifert H, Chen L et al. Standard and higher dose of olanzapine in patients with schizophrenia or schizoaffective disorder: a randomized, double-blind, fixed-dose study. / Clin Psychopharmacol 2008; 28: 392-400.

13 Rorick-Kehn LM, Johnson BG, Burkey JL, Wright RA, Calligaro DO, Marek GJ et al. Pharmacological and pharmacokinetic properties of a structurally novel, potent, and selective metabotropic glutamate 2/3 receptor agonist: in vitro characterization of agonist (-)-(1R,4S,5S,6S)4-amino-2-sulfonylbicyclo[3.1.0]-hexane-4,6-dicarboxylic acid (LY404039). J Pharmacol Exp Ther 2007; 321: 308-317.

14 Bymaster F, Perry KW, Nelson DL, Wong DT, Rasmussen K, Moore NA et al. Olanzapine: a basic science update. Br J Psychiatry Suppl 1999; 37: 36-40.

15 Muller DJ, Kennedy JL. Genetics of antipsychotic treatment emergent weight gain in schizophrenia. Pharmacogenomics 2006; 7: 863-887.

16 Mei L, Xiong WC. Neuregulin 1 in neural development, synaptic plasticity and schizophrenia. Nat Rev Neurosci 2008; 9: 437-452.

17 Gupta M, Bhatnagar P, Grover S, Kaur H, Baghel R, Bhasin Y et al. Association studies of catechol-O-methyltransferase (COMT) gene with schizophrenia and response to antipsychotic treatment. Pharmacogenomics 2009; 10: 385-397.

18 Chen Q, He G, Chen Q, Wu S, Xu Y, Feng G et al. A case-control study of the relationship between the metabotropic glutamate receptor 3 gene and schizophrenia in the Chinese population. Schizophr Res 2005; 73: 21-26.

19 Egan MF, Straub RE, Goldberg TE, Yakub I, Callicott JH, Hariri AR et al. Variation in GRM3 affects cognition, prefrontal glutamate, and risk for schizophrenia. Proc Natl Acad Sci USA 2004; 101: 12604-12609.

20 Fujii Y, Shibata H, Kikuta R, Makino C, Tani A, Hirata N et al. Positive associations of polymorphisms in the metabotropic glutamate receptor type 3 gene (GRM3) with schizophrenia. Psychiatr Genet 2003; 13: $71-76$.

21 Jurinke $\mathrm{C}$, van den Boom D, Cantor CR, Koster $\mathrm{H}$. Automated genotyping using the DNA MassArray technology. Methods $\mathrm{Mol} B \mathrm{Biol}$ 2001; 170: 103-116.

22 Benjamini $Y$, Hochberg $Y$. Controlling the false discovery rate: a practical and powerful approach to multiple testing. I $R$ Stat Soc Series B Stat Methodol 1995; 57: 289-300.

23 Barrett JC, Fry B, Maller J, Daly MJ. Haploview: analysis and visualization of LD and haplotype maps. Bioinformatics 2005; 21: 263-265.

24 Peng G, Luo L, Siu H, Zhu Y, Hu P, Hong S et al. Gene and pathwaybased second wave analysis of genome-wide association studies. Eur I Hum Genet 2010; 18: 111-117.

25 Li D, Collier DA, He L. Meta-analysis shows strong positive association of the neuregulin 1 (NRG1) gene with schizophrenia. Hum Mol Genet 2006; 15: 1995-2002.

26 Wang F, Jiang T, Sun Z, Teng SL, Luo X, Zhu Z et al. Neuregulin 1 genetic variation and anterior cingulum integrity in patients with schizophrenia and healthy controls. J Psychiatry Neurosci 2009; 34: 181-186.

27 Cartmell J, Perry KW, Salhoff CR, Monn JA, Schoepp DD. The potent, selective mGlu2/3 receptor agonist LY379268 increases extracellular levels of dopamine, 3,4-dihydroxyphenylacetic acid, homovanillic acid, 
and 5-hydroxyindole-3-acetic acid in the medial prefrontal cortex of the freely moving rat. J Neurochem 2000; 75: 1147-1154.

28 Rorick-Kehn LM, Johnson BG, Knitowski KM, Salhoff CR, Witkin JM, Perry KW et al. In vivo pharmacological characterization of the structurally novel, potent, selective mGlu2/3 receptor agoinst LY404039 in animal models of psychiatric disorders. Psychopharmacology (Berl) 2007; 193: 121-136.

29 Zhang Y, Bertolino A, Fazio L, Blasi G, Rampino A, Romano R et al. Polymorphisms in human dopamine D2 receptor gene affect gene expression, splicing, and neuronal activity during working memory. Proc Natl Acad Sci USA 2007; 104: 20552-20557.

30 Bertolino A, Blasi G. The genetics of schizophrenia. Neuroscience 2009; 164: 288-299.

31 Centonze D, Grande C, Usiello A, Gubellini P, Erbs E, Martin AB et al Receptor subtypes involved in the presynaptic and postsynaptic actions of dopamine on striatal interneurons. I Neurosci 2003; 23: 6245-6254.

32 Centonze D, Gubellini P, Usiello A, Rossi S, Tscherter A, Bracci E et al Differential contribution of dopamine D2S and D2L receptors in the modulation of glutamate and GABA transmission in the striatum. Neuroscience 2004; 129: 157-166.

33 Marek G), Wright RA, Schoepp DD. 5-Hydroxytryptamine2A (5-HT2A) receptor regulation in rat prefrontal cortex: interaction of a phenethy lamine hallucinogen and the metabotropic glutamate2/3 receptor agonist LY354740. Neurosci Lett 2006; 403: 256-260.
34 Gonzalez-Maeso J, Ang RL, Yuen T, Chan P, Weisstaub NV, Lopez-Gimenez JF et al. Identification of a serotonin/glutamate receptor complex implicated in psychosis. Nature 2008; 452: 93-97.

35 The ENCODE Project Consortium. Identification and analysis of functional elements in $1 \%$ of the human genome by the ENCODE pilot project. Nature 2007; 447: 799-816.

36 Yelin R, Dahary D, Sorek R, Levanon EY, Goldstein O, Shoshan A et al. Widespread occurrence of antisense transcription in the human genome. Nat Biotechnol 2003; 21: 379-386.

37 Werner A, Sayer JA. Naturally occurring antisense RNA: function and mechanisms of action. Curr Opin Nephrol Hypertens 2009; 18: 343-349.

38 Patrick DL, Burns T, Morosini P, Rothman M, Gagnon DD, Wild D et al. Reliability, validity and ability to detect change of the clinician-rated Personal and Social Performance scale in patients with acute symptoms of schizophrenia. Curr Med Res Opin 2009; 25: 325-338.

This work is licensed under the Creative Commons Attribution-NonCommercial-No Derivative Works 3.0 Unported License. To view a copy of this license, visit http://creativecommons.org/ licenses/by-nc-nd/3.0/

Supplementary Information accompanies the paper on the The Pharmacogenomics Journal website (http://www.nature.com/tpj) 